\title{
LATTICE RULES: PROJECTION REGULARITY AND UNIQUE REPRESENTATIONS
}

\author{
I. H. SLOAN AND J. N. LYNESS
}

\begin{abstract}
We introduce a unique characterization for lattice rules which are projection regular. Any such rule, having invariants $n_{1}, n_{2}, \ldots, n_{s}$, may be expressed, uniquely, in the form

$$
Q f=\frac{1}{n_{1} n_{2} \cdots n_{s}} \sum \sum \cdots \sum \bar{f}\left(\frac{j_{1} \mathbf{z}_{1}}{n_{1}}+\frac{j_{2} \mathbf{z}_{2}}{n_{2}}+\cdots+\frac{j_{s} \mathbf{z}_{s}}{n_{s}}\right),
$$

where the matrix $Z=\left(\mathbf{z}_{1}, \mathbf{z}_{2}, \ldots, \mathbf{z}_{s}\right)^{T}$ is upper unit triangular and individual elements satisfy $0 \leq z_{r}^{(c)}<\left(n_{r} / n_{c}\right), r<c$.
\end{abstract}

\section{INTRODUCTION}

The notion of a lattice rule for numerical integration over the unit $s$-dimensional cube was introduced in Sloan [4] and Sloan and Kachoyan [5], and further discussed in Sloan and Lyness [6], where it was shown that any $s$-dimensional lattice rule $Q_{s}$ can be expressed in the canonical form

$$
Q_{s} f=\frac{1}{n_{1} n_{2} \cdots n_{s}} \sum_{j_{1}=1}^{n_{1}} \sum_{j_{2}=1}^{n_{2}} \cdots \sum_{j_{s}=1}^{n_{s}} \bar{f}\left(\sum_{i=1}^{s} \frac{j_{i} \mathbf{z}_{i}}{n_{i}}\right) .
$$

Here the invariants $n_{1}, n_{2}, \ldots, n_{s}$ are positive integers satisfying

$$
n_{i+1} \mid n_{i}, \quad i=1, \ldots, s-1
$$

$\mathbf{z}_{i} \in \mathbb{Z}^{s}$ for $i=1, \ldots, s$; and $\bar{f}$ is a 1-periodic extension of $f$. (For a more precise specification of $\bar{f}$ see Sloan and Lyness [6].) The abscissa set of the rule $Q_{s}$ is the set

$$
A\left(Q_{s}\right)=\left\{\left\{\sum_{i=1}^{s} \frac{j_{i} \mathbf{z}_{i}}{n_{i}}\right\}: j_{i}=1, \ldots, n_{i}, i=1, \ldots, s\right\},
$$

where $\{\mathbf{v}\}$ denotes the vector whose components are the fractional parts of those of $\mathbf{v}$. Clearly, $\{\mathbf{v}\} \in[0,1)^{s}$, the half-open unit cube. The order of $Q_{s}$ is

Received March 27, 1989.

1980 Mathematics Subject Classification (1985 Revision). Primary 65D30.

This work was supported in part by the Applied Mathematical Sciences subprogram of the Office of Energy Research, U. S. Department of Energy, under Contract W-31-109-Eng-38, and in part by the Australian Research Council. 
the number $\nu\left(Q_{s}\right)$ of distinct elements of the abscissa set; since the abscissa set (1.3) of a rule (1.1) in canonical form is by definition nonrepetitive, the order is $\nu\left(Q_{s}\right)=n_{1} n_{2} \cdots n_{s}$.

The invariants in (1.1) are uniquely defined, but there is a wide choice available for the vectors $\mathbf{z}_{1}, \mathbf{z}_{2}, \ldots, \mathbf{z}_{s}$. The purpose of this paper is to show that for a particular class of lattice rules a representation of the form (1.1) can be prescribed in which even the vectors $\mathbf{z}_{1}, \mathbf{z}_{2}, \ldots, \mathbf{z}_{s}$ are uniquely determined.

The rules we consider have principal projections that are well behaved in a certain sense. The $d$-dimensional principal projection of $Q_{s}$ is the rule $Q_{d}$ obtained by retaining only the first $d$ components of each abscissa. In Lyness and Sloan [1] it is shown that, in terms of the invariants of $Q_{s}$, its principal projection $Q_{d}$ has $\nu\left(Q_{d}\right) \leq n_{1} n_{2} \cdots n_{d}$ distinct abscissas. A rule $Q_{s}$ for which equality is achieved for every principal projection, that is for which

$$
\nu\left(Q_{d}\right)=n_{1} n_{2} \cdots n_{d}, \quad d=1,2, \ldots, s,
$$

is said to be projection regular. Thus a projection regular rule is one in which each principal projection has as many distinct points as possible for a rule with the given invariants. Projection regular rules are the focus of this paper.

The Z-matrix of the canonical form (1.1) is the $s \times s$ matrix defined by

$$
Z=\left(\mathbf{z}_{1}, \mathbf{z}_{2}, \ldots, \mathbf{z}_{s}\right)^{T} \text {. }
$$

We shall establish the following characterization of projection regular rules.

Theorem 1.1. A lattice rule $Q_{s}$ is projection regular if and only if there exists a canonical form for which the Z-matrix is upper unit triangular.

This result will lead us to a standard form for projection regular rules, in which not only is the $Z$-matrix upper unit triangular, but also all elements of the $Z$-matrix are uniquely determined.

In $\S 2$ we collect together transformations of the $Z$-matrix which leave the rule unaltered. In $\S 3$ principal projections are defined, and we establish the easier part of the above theorem, namely that if $Z$ is upper unit triangular, then $Q_{s}$ is projection regular, while $\S 4$ is devoted to the more difficult converse. In $\S 5$ we use the results to establish the promised standard form for projection regular rules. The paper concludes with a brief discussion in $\S 6$.

\section{TRANSFORMATIONS OF THE $Z$-MATRIX}

We now consider a more general (possibly repetitive) form of the lattice rule $Q_{s}$,

$$
Q_{s} f=\frac{1}{n_{1} n_{2} \cdots n_{t}} \sum_{j_{1}=1}^{n_{1}} \sum_{j_{2}=1}^{n_{2}} \cdots \sum_{j_{t}=1}^{n_{t}} \bar{f}\left(\sum_{i=1}^{t} \frac{j_{i} \mathbf{z}_{i}}{n_{i}}\right),
$$

where $t$ and $n_{1}, n_{2}, \ldots, n_{t}$ are positive integers (not necessarily satisfying the property (1.2)), and $\mathbf{z}_{i} \in \mathbb{Z}^{s}, i=1, \ldots, s$. In this more general situation the 
$Z$-matrix is a $t \times s$ matrix,

$$
Z=\left(\mathbf{z}_{1}, \mathbf{z}_{2}, \ldots, \mathbf{z}_{t}\right)^{T}
$$

Our concern in this section is to find transformations which change $Z$ to a new matrix $Z^{\prime}$,

$$
Z^{\prime}=\left(\mathbf{z}_{1}^{\prime}, \mathbf{z}_{2}^{\prime}, \ldots, \mathbf{z}_{t}^{\prime}\right)^{T}
$$

while leaving the rule $Q_{s}$ unchanged. Throughout this section the numbers $t, n_{1}, \ldots, n_{t}$ are considered fixed.

Given the rule $Q_{s}$ in the form (2.1), it is convenient to introduce

$$
\mathbf{c}_{i}=\mathbf{z}_{i} / n_{i}, \quad i=1,2, \ldots, t .
$$

Then the lattice corresponding to this rule (see Sloan and Kachoyan [5]) can be written

$$
L=\left\{\sum_{i=1}^{t} j_{i} \mathbf{c}_{i}+\mathbf{k}: j_{i} \in \mathbb{Z} \text { for } i=1, \ldots, t \text {, and } \mathbf{k} \in \mathbb{Z}^{s}\right\} .
$$

Note that $L \cap[0,1)^{s}=A\left(Q_{s}\right)$.

It is clear that a given transformation of the $Z$-matrix leaves the rule $Q_{s}$ unchanged if, and only if, it leaves the corresponding lattice $L$ unchanged.

Transformations which leave the lattice $L$, defined by $(2.5)$, unchanged are: for given $i \in\{1,2, \ldots, t\}$, replace $\mathbf{c}_{i}$ by

$$
\mathbf{c}_{i}^{\prime}=-\mathbf{c}_{i}
$$

or by

$$
\mathbf{c}_{i}^{\prime}=\mathbf{c}_{i}+\mathbf{k}, \quad \text { where } \mathbf{k} \in \mathbb{Z}^{s}
$$

or by

$$
\mathbf{c}_{i}^{\prime}=\mathbf{c}_{i}+\mathbf{c}_{l}, \quad \text { where } l \neq i
$$

It can be shown that all the affine transformations that leave $L$ unchanged are combinations of these three.

We are interested only in those transformations which retain the form (2.4), i.e., with $n_{i}$ being given. We require in addition the conditions

$$
\mathbf{z}_{i}:=n_{i} \mathbf{c}_{i} \in \mathbb{Z}^{s}, \quad \mathbf{z}_{i}^{\prime}:=n_{i} \mathbf{c}_{i}^{\prime} \in \mathbb{Z}^{s}, \quad i=1,2, \ldots, s .
$$

The following theorem includes all these.

Theorem 2.1. The rule $Q_{s}$ given by equation (2.1) is unchanged if for given $i \in$ $\{1,2, \ldots, t\}$ the vector $\mathbf{z}_{i}$ is replaced by any of
(a) $\mathbf{z}_{i}^{\prime}=r \mathbf{z}_{i}$ if $r \in \mathbb{Z}$ and $r$ and $n_{i}$ are relatively prime;
(b) $\mathbf{z}_{i}^{\prime}=\mathbf{z}_{i}+n_{i} \mathbf{k}$, where $\mathbf{k} \in \mathbb{Z}^{s}$;
(c) $\mathbf{z}_{i}^{\prime}=\mathbf{z}_{i}+n n_{i} \mathbf{z}_{l} / n_{l}$, where $l \neq i, \quad n \in \mathbb{Z}$, and $n_{l} \mid n n_{i}$. 
Proof. Parts (b) and (c) follow immediately from (2.7) and (2.8), with the second condition in (c) coming from (2.4), or, equivalently, from the requirement that $\mathbf{z}_{i}, \mathbf{z}_{i}^{\prime} \in \mathbb{Z}^{s}$. Part (a) is more interesting. Note that it subsumes (2.6) when $r=-1$. Since $r$ and $n_{i}$ are relatively prime, it is well known that

$$
\left\{r, 2 r, \ldots, n_{i} r\right\}=\left\{1,2, \ldots, n_{i}\right\} \quad\left(\bmod n_{i}\right) .
$$

It follows that $\left\{\left\{j r z_{i} / n_{i}\right\}: j=1,2, \ldots, n_{i}\right\}$ is merely a reordering of $\left\{\left\{j \mathbf{z}_{i} / n_{i}\right\}: j=1,2, \ldots, n_{i}\right\}$, so that the rule $(2.1)$ is unchanged.

In the important special case in which $Q_{s}$ is given in canonical form (1.1) we have, for each pair of invariants $n_{i}, n_{l}$, either $n_{l} \mid n_{i}$ or $n_{i} \mid n_{l}$ (or both, if $n_{l}=n_{i}$ ). There is therefore good reason to be interested in the following special cases of Theorem 2.1(c).

Corollary 2.2. The rule $Q_{s}$ given by (2.1) is unchanged if the vector $\mathbf{z}_{i}$ is replaced by

(a) $\mathbf{z}_{i}^{\prime}=\mathbf{z}_{i}+n\left(n_{i} / n_{l}\right) \mathbf{z}_{l}$ if $n_{l} \mid n_{i}, \quad l \neq i$, and $n \in \mathbb{Z}$;

(b) $\mathbf{z}_{i}^{\prime}=\mathbf{z}_{i}+k \mathbf{z}_{l}$ if $n_{i} \mid n_{l}, \quad l \neq i$, and $k \in \mathbb{Z}$.

\section{Projection regular Rules}

We begin with a definition from Lyness and Sloan [1].

Definition. The $d$-dimensional principal projection of an $s$-dimensional quadrature rule for the unit cube is the $d$-dimensional rule obtained by omitting the final $s-d$ components of each abscissa.

Specifically, the $d$-dimensional principal projection of the quadrature rule

$$
Q_{s} f=\sum_{j=1}^{N} w_{j} f\left(\zeta_{j}^{(1)}, \zeta_{j}^{(2)}, \ldots, \zeta_{j}^{(s)}\right)
$$

is

$$
Q_{d} f=\sum_{j=1}^{N} w_{j} f\left(\zeta_{j}^{(1)}, \zeta_{j}^{(2)}, \ldots \zeta_{j}^{(d)}\right)
$$

The $d$-dimensional principal projection $Q_{d}$ of a lattice rule $Q_{s}$ is itself a lattice rule (see Sloan and Lyness [6, Theorem 5.1]). This leads to the following characterization of $Q_{d}$.

Theorem 3.1. If $Q_{s}$ is an s-dimensional lattice rule, then its d-dimensional principal projection $Q_{d}$ is characterized by the three properties:

(a) $Q_{d}$ is a d-dimensional lattice rule;

(b) $\left(\zeta^{(1)}, \zeta^{(2)}, \ldots, \zeta^{(s)}\right) \in A\left(Q_{s}\right) \Rightarrow\left(\zeta^{(1)}, \zeta^{(2)}, \ldots, \zeta^{(d)}\right) \in A\left(Q_{d}\right)$;

(c) $\left(\zeta^{(1)}, \zeta^{(2)}, \ldots, \zeta^{(d)}\right) \in A\left(Q_{d}\right) \Rightarrow$ there exists an element of $A\left(Q_{s}\right)$ of the form

$$
\left(\zeta^{(1)}, \zeta^{(2)}, \ldots, \zeta^{(d)}, x^{(d+1)}, \ldots, x^{(s)}\right)
$$


Proof. This is almost self-evident: given $Q_{s}$, abscissas of $Q_{d}$ are determined by (b). Additional abscissas are excluded by (c). Since $Q_{d}$ is a lattice rule, each abscissa has the same weight. Thus $Q_{d}$ is determined by (a), (b), and (c).

Theorem 3.2. If $Q_{s}$ is given by (1.1), with $n_{1}, \ldots, n_{s}$ satisfying (1.2) and the $Z$-matrix in upper unit triangular form, then

(a) $Q_{s}$ is in canonical form; and

(b) for $d \in\{1,2, \ldots, s\}$ the rule

$$
Q_{d} f=\frac{1}{n_{1} n_{2} \cdots n_{d}} \sum_{j_{1}=1}^{n_{1}} \sum_{j_{2}=1}^{n_{2}} \cdots \sum_{j_{d}=1}^{n_{d}} \bar{f}\left(\sum_{i=1}^{d} \frac{j_{i}\left(z_{i}^{(1)}, z_{i}^{(2)}, \ldots, z_{i}^{(d)}\right)}{n_{i}}\right)
$$

is the d-dimensional principal projection of $Q_{s} f$, expressed in canonical form.

Proof. (a) That (1.1) is nonrepetitive when the $Z$-matrix is upper unit triangular follows easily by inspecting the abscissa set $A\left(Q_{s}\right)$ : for each value of $k=1,2, \ldots, s$ it contains the $n_{k}$ distinct points

$$
\left\{\left\{j_{k} \frac{\left(0,0, \ldots, 0,1, z_{k}^{(k+1)}, \ldots, z_{k}^{(s)}\right)}{n_{k}}\right\}: J_{k}=1,2, \ldots, n_{k}\right\}
$$

(where the 1 occurs in the $k$ th component), obtained from (1.1) by setting $j_{i}=0$ for $i \neq k$. The points obtained for different values of $k$ are distinct. Thus there are $n_{1} n_{2} \cdots n_{s}$ distinct points in $A\left(Q_{s}\right)$, and (1.1) is nonrepetitive.

(b) Almost by inspection, $Q_{d}$ given by (3.1) satisfies the conditions of Theorem 3.1 with respect to $Q_{s}$. Thus it is indeed the principal projection of $Q_{s}$. Finally, since $Q_{d}$ is in the same form as $Q_{s}$, but with $d$ replacing $s$, and since $Q_{d}$ has an upper unit triangular $Z$-matrix, it follows from (a) with $s$ replaced by $d$ that (3.1) is the canonical form of $Q_{d}$.

Since (3.1) is in canonical form, we have $\nu\left(Q_{d}\right)=n_{1} n_{2} \cdots n_{d}$, from which it follows, by the definition in the Introduction, that $Q_{s}$ is projection regular. Thus Theorem 3.2 yields:

Theorem 3.3. If $Q_{s}$ is given by (1.1), with $n_{1}, n_{2}, \ldots, n_{s}$ satisfying (1.2) and the $Z$-matrix in upper unit triangular form, then $Q_{s}$ is projection regular.

\section{THE CONVERSE RESULT}

In this section we establish:

Theorem 4.1. If the lattice rule $Q_{s}$ is projection regular, it may be expressed in canonical form with the Z-matrix upper unit triangular.

This is the converse of Theorem 3.3. Taken together, these give Theorem 1.1 stated in the Introduction.

Throughout this section, $n_{1}, n_{2}, \ldots, n_{s}$ denote the invariants of $Q_{s}$; thus

$$
n_{i+1} \mid n_{i}, \quad i=1,2, \ldots, s-1,
$$


and there exists $Z=\left(\mathbf{z}_{1}, \mathbf{z}_{2}, \ldots, \mathbf{z}_{s}\right)^{T}$ such that

$$
Q_{s} f=\frac{1}{n_{1} n_{2} \cdots n_{s}} \sum_{j_{1}=1}^{n_{1}} \sum_{j_{2}=1}^{n_{2}} \cdots \sum_{j_{s}=1}^{n_{s}} \bar{f}\left(\sum_{r=1}^{s} \frac{j_{r} \mathbf{z}_{r}}{n_{r}}\right)
$$

is in canonical form. Moreover, because $Q_{s}$ is projection regular, the $d$ dimensional principal projection $Q_{d}$ has invariants $n_{1}, n_{2}, \ldots, n_{d}$, and has order

$$
\nu\left(Q_{d}\right)=n_{1} n_{2} \cdots n_{d} .
$$

Our proof of Theorem 4.1 is by induction on the columns of the $Z$-matrix, the inductive step being:

Lemma 4.2. Let $d$ be an integer satisfying $1 \leq d \leq s$. If the projection regular rule $Q_{s}$ can be expressed in the canonical form (4.1) with a Z-matrix whose first $d-1$ columns are in upper unit triangular form, then it may also be expressed in canonical form with a Z-matrix whose first $d$ columns are in upper unit triangular form.

Proof of Lemma 4.2. It is convenient to denote the $d$ th column of the $Z$-matrix, i.e., the column currently being treated, by $\zeta$, so that $\zeta_{r}=z_{r}^{(d)}, r=1, \ldots, s$. Then the $d$-dimensional principal projection of $(4.1)$ is given by

$$
Q_{d} f=\frac{1}{n_{1} n_{2} \cdots n_{s}} \sum_{j_{1}=1}^{n_{1}} \sum_{j_{2}=1}^{n_{2}} \cdots \sum_{j_{s}=1}^{n_{s}} \bar{f}\left(\sum_{r=1}^{s} \frac{j_{r}\left(z_{r}^{(1)}, \ldots, z_{r}^{(d-1)}, \zeta_{r}\right)}{n_{r}}\right) .
$$

The hypothesis of the lemma is that the elements of the $Z$-matrix in (4.1) satisfy

$$
z_{r}^{(c)}=\delta_{r c} \quad \text { for } r=c, c+1, \ldots, s, c=1,2, \ldots, d-1 .
$$

We plan to provide a set of transformations which transform the $Z$-matrix, without changing $Q_{s}$, in such a way that (4.4) continues to hold, but in addition

$$
\zeta_{d}=z_{d}^{(d)}=1
$$

and

$$
\zeta_{r}=z_{r}^{(d)}=0 \text { for } r=d+1, d+2, \ldots, s .
$$

We shall specify sets of transformations ((4.11) and (4.16) below) which, while leaving the first $d-1$ columns of the $Z$-matrix unchanged, produce a value of $\zeta_{d}$ satisfying (4.5), and after this a second set ((4.18) below) which set the $\zeta_{r}$ of $(4.6)$ to zero. However, before establishing these transformations we need the following:

Lemma 4.3. Under the hypotheses of Lemma 4.2 the quantities in (4.3) satisfy

$$
\left(\zeta_{d}, \frac{n_{d}}{n_{d+1}} \zeta_{d+1}, \ldots, \frac{n_{d}}{n_{s}} \zeta_{s}, n_{d}\right)=1,
$$

where, as before, $\zeta_{r}$ stands for $z_{r}^{(d)}$. 
The quantity on the left-hand side of (4.7) is the greatest common divisor of the numbers separated by commas.

We now prove Lemma 4.3. Because $Q_{s}$ is projection regular, the abscissa set $A\left(Q_{d}\right)$ is of order $n_{1} n_{2} \cdots n_{d}$. We are interested in the subgroup $S_{d}$ of $A\left(Q_{d}\right)$ obtained by setting $j_{1}=j_{2}=\cdots=j_{d-1}=0$ in (4.3). Because the first $d-1$ columns of the $Z$-matrix are already in upper unit triangular form, we obtain from (4.3)

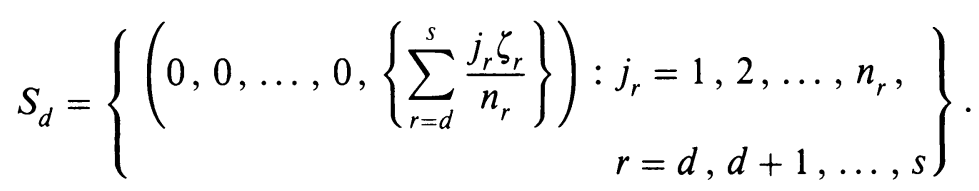

The $d$ th components of (4.8) form the abscissa set of a one-dimensional lattice rule. Since the only such rule is the trapezoidal rule, the only thing not yet known is the spacing. We assert that $S_{d}$ contains exactly $n_{d}$ distinct points, and therefore

$$
S_{d}=\left\{\left(0,0, \ldots, 0, \frac{j}{n_{d}}\right): j=0,1, \ldots, n_{d}-1\right\} .
$$

To show this, first observe that the summations over $j_{1}, \ldots, j_{d-1}$ in (4.3), with $j_{d}, \ldots, j_{s}$ set equal to $n_{d}, \ldots, n_{s}$ respectively, give exactly $n_{1} n_{2} \cdots n_{d-1}$ distinct abscissas; this is easily verified directly, given that the first $d-1$ columns of the $Z$-matrix are already in upper unit triangular form. Since $\nu\left(Q_{d}\right)=n_{1} n_{2} \cdots n_{d}$, the remaining sums, with $j_{1}, \ldots, j_{d-1}$ set to zero, must yield exactly $n_{d}$ abscissas. Thus (4.9) is established.

It follows from the equivalence of (4.8) and (4.9) that there must exist integers $j_{d}, j_{d+1}, \ldots, j_{s}$ and $l$ satisfying $1 \leq j_{r} \leq n_{r}$ such that

$$
\frac{1}{n_{d}}=j_{d} \frac{\zeta_{d}}{n_{d}}+j_{d+1} \frac{\zeta_{d+1}}{n_{d+1}}+\cdots+j_{s} \frac{\zeta_{s}}{n_{s}}+l
$$

or equivalently

$$
1=j_{d} \zeta_{d}+j_{d+1} \frac{n_{d}}{n_{d+1}} \zeta_{d+1}+\cdots+j_{s} \frac{n_{d}}{n_{s}} \zeta_{s}+\ln n_{d}
$$

Any factor shared by each term on the right must occur in the left. This establishes Lemma 4.3.

Returning to Lemma 4.2, we are now ready to construct a sequence of transformations of the $Z$-matrix which change only the $d$ th row of the matrix, and leave all other rows unchanged. We shall exploit Lemma 4.3 above to show these give $z_{d}^{(d)}=1$. The transformations consist of row operations of the form

$$
\mathbf{z}_{d}(i)=\mathbf{z}_{d}(i-1)+k_{i} \frac{n_{d}}{n_{i}} \mathbf{z}_{i}, \quad i=d+1, \ldots, s,
$$

where the initial vector $\mathbf{z}_{d}(d)$ is the $d$ th row of the $Z$-matrix as given to us in the hypotheses of Lemma 4.2. Note that the leading $d-1$ zeros in 
$\mathbf{z}_{d}$ are not altered by the transformations, and that by Corollary $72.2(\mathrm{a})$ the transformations (4.11) do not change $Q_{s}$.

Consistently with our earlier notation, we denote the $d$ th component of $\mathbf{z}_{d}(i)$ by $\zeta_{d}(i)$, so that the leading nonzero component of (4.11) takes the form

$$
\zeta_{d}(i)=\zeta_{d}(i-1)+k_{i} \frac{n_{d}}{n_{i}} \zeta_{i}, \quad i=d+1, \ldots, s .
$$

We are now ready to specify $k_{i}$ in (4.11): it is chosen so that

$$
\left(\zeta_{d}(i-1)+k_{i} \frac{n_{d}}{n_{i}} \zeta_{i}, n_{d}\right)=\left(\zeta_{d}(i-1), \frac{n_{d}}{n_{i}} \zeta_{i}, n_{d}\right),
$$

the existence of an integer with this property being guaranteed by Lemma A1 in the appendix. Since (4.12) and (4.13) imply

$$
\left(\zeta_{d}(i), n_{d}\right)=\left(\zeta_{d}(i-1), \frac{n_{d}}{n_{i}} \zeta_{i}, n_{d}\right), \quad i=d+1, \ldots, s,
$$

it follows recursively that at the end of the sequence of transformations we have

$$
\left(\zeta_{d}(s), n_{d}\right)=\left(\zeta_{d}(d), \frac{n_{d}}{n_{d+1}} \zeta_{d+1}, \ldots, \frac{n_{d}}{n_{s}} \zeta_{s}, n_{d}\right),
$$

and hence, from Lemma 4.3,

$$
\left(\zeta_{d}(s), n_{d}\right)=1
$$

Let us now denote by $\zeta_{d}$ instead of $\zeta_{d}(s)$ the value of $z_{d}^{(d)}$ achieved at the end of the sequence of transformations (4.11). Since $\left(\zeta_{d}, n_{d}\right)=1$, there exists an integer $q$ such that

$$
q \zeta_{d} \equiv 1 \quad\left(\bmod n_{d}\right)
$$

Since $\left(q, n_{d}\right)=1$, the transformation

$$
\mathbf{z}_{d}^{\prime}=q \mathbf{z}_{d}
$$

leaves $Q_{s}$ unchanged by Theorem 2.1(a), and at the same time ensures

$$
\zeta_{d}^{\prime}:=z_{d}^{(d)} \equiv 1 \quad\left(\bmod n_{d}\right) .
$$

An application of Theorem 2.1(b) then allows us to replace $\zeta_{d}^{\prime}$ by 1 without changing $Q_{s}$. We now have the value of $\zeta_{d}=z_{d}^{(d)}$ required in (4.5).

A final set of transformations

$$
\mathbf{z}_{r}^{\prime}=\mathbf{z}_{r}-\zeta_{r} \mathbf{z}_{d}, \quad r=d+1, \ldots, s
$$

(where $\mathbf{z}_{d}$ denotes the current $d$ th row of the $Z$-matrix, satisfying (4.5)), now replaces the elements $z_{r}^{(d)}=\zeta_{r}$ in the lower part of the $d$ th column of the $Z$-matrix by zeros, while at the same time, by Corollary 2.2(b), leaves $Q_{s}$ unaltered. The proof of Lemma 4.2 is now complete.

Theorem 4.1 now follows from Lemma 4.2 by induction on $d$. 
5. A UNIQUE REPRESENTATION FOR PROJECTION REGULAR LATTICE RULES

In this section we establish a unique standard form for all projection regular lattice rules.

Definition. $Q_{s}$ of $(1.1)$ is in standard form when the elements of its $Z$-matrix satisfy

$$
\begin{gathered}
z_{r}^{(c)}=0, \quad 1 \leq c<r \leq s, \\
z_{c}^{(c)}=1, \quad c=1,2, \ldots, s, \\
0 \leq z_{r}^{(c)}<n_{r} / n_{c}, \quad 1 \leq r<c \leq s .
\end{gathered}
$$

Thus $Z$ is an upper unit triangular matrix and so, from Theorem 3.3, a rule $Q_{s}$ in standard form must be a projection regular rule.

In this section we establish

Theorem 5.1. A projection regular lattice rule with invariants $n_{1}, n_{2}, \ldots, n_{s}$ can be expressed, uniquely, in standard form.

To establish the theorem, we use Theorem 4.1, and also prove in turn Lemmas 5.2 and 5.3 below. The latter deals with uniqueness.

Lemma 5.2. When $Q_{s}$ is given in canonical form (1.1) with upper unit triangular $Z$-matrix, it may be re-expressed with a Z-matrix in standard form.

Proof. This can be effected by transformations of the form

$$
\mathbf{z}_{r}^{\prime}=\mathbf{z}_{r}-\left[\frac{z_{r}^{(c)} n_{c}}{n_{r}}\right] \frac{n_{r}}{n_{c}} \mathbf{z}_{c} \quad \text { for } r<c,
$$

which according to Corollary 2.2(a) leave $Q_{s}$ unaffected. The transformation (5.3) affects only the $r$ th row $\mathbf{z}_{r}$ of the matrix. Since $\mathbf{z}_{c}$ has $c-1$ leading zero components, it leaves unaltered the first $c-1$ elements of $z_{r}$, and generally alters the other elements. In particular, since $z_{c}^{(c)}=1$, it replaces $z_{r}^{(c)}$ by

$$
z_{r}^{\prime(c)}=z_{r}^{(c)}-\left[\frac{z_{r}^{(c)} n_{c}}{n_{r}}\right] \frac{n_{r}}{n_{c}}
$$

which satisfies (5.2). It is necessary to carry out these transformations in an order arranged so that, once $z_{r}^{(c)}$ has been replaced by $z_{r}^{\prime(c)}$, the new element is not affected by any subsequent transformation. It is readily verified that a suitable order is obtained so long as the transformation involving $z_{r}^{(c)}$ is carried out only after all transformations involving a lower column number $c^{\prime}<c$ are completed. This establishes Lemma 5.2.

Lemma 5.3. The rule $Q_{s}$ may be represented in standard form in only one way. Proof. Suppose that $Z=\left(\mathbf{z}_{1}, \mathbf{z}_{2}, \ldots, \mathbf{z}_{s}\right)^{T}$ and $W=\left(\mathbf{w}_{1}, \mathbf{w}_{2}, \ldots, \mathbf{w}_{s}\right)^{T}$ are two alternative $Z$-matrices for the rule $Q_{s}$, both in standard form, whose first 
$d-1$ columns coincide. Given $r \in\{1,2, \ldots, d-1\}$, the vectors $\mathbf{z}_{r} / n_{r}$ and $\mathbf{w}_{r} / n_{r}$ belong to the lattice corresponding to $Q_{s}$, and therefore so too does $\left(\mathbf{z}_{r}-\mathbf{w}_{r}\right) / n_{r}$. On retaining only the leading $d$ components of each vector, we conclude that the $d$-vector

$$
\left(0,0, \ldots, 0, \frac{z_{r}^{(d)}-w_{r}^{(d)}}{n_{r}}\right)
$$

belongs to the lattice corresponding to the $d$-dimensional principal projection $Q_{d}$. From this it follows that

$$
\left(0,0, \ldots, 0, \frac{z_{r}^{(d)}-w_{r}^{(d)}}{n_{r}}\right)=\sum_{i=1}^{d} j_{i} \frac{\left(z_{i}^{(1)}, z_{i}^{(2)}, \ldots, z_{i}^{(d)}\right)}{n_{i}} \quad(\bmod 1)
$$

for some integers $j_{1}, j_{2}, \ldots, j_{d}$. Because of the upper unit triangular nature of $Z$, it is easily seen that this can be satisfied only if

$$
j_{i} \equiv 0 \quad\left(\bmod n_{i}\right), \quad i=1,2, \ldots, d-1 ;
$$

thus we conclude that

$$
\left(0,0, \ldots, 0, \frac{z_{r}^{(d)}-w_{r}^{(d)}}{n_{r}}\right)=j_{d} \frac{\left(0,0, \ldots, 0, z_{d}^{(d)}\right)}{n_{d}}=j_{d} \frac{(0, \ldots, 0,1)}{n_{d}}
$$

or

$$
z_{r}^{(d)}-w_{r}^{(d)}=j_{d} \frac{n_{r}}{n_{d}}
$$

for some integer $j_{d}$. Thus $z_{r}^{(d)}$ differs from $w_{r}^{(d)}$ by some integer multiple of $n_{r} / n_{d}$. Since by (5.2) both $z_{r}^{(d)}$ and $w_{r}^{(d)}$ are in the interval $\left[0, n_{r} / n_{d}\right)$, it follows that they coincide.

We recall that our hypothesis is that the first $d-1$ columns of $Z$ and $W$ coincide. The argument above shows that all elements of the $d$ th column also coincide, and Lemma 5.3 then follows by induction.

Lemmas 5.2 and 5.3 together with Theorem 4.1 establish Theorem 5.1.

\section{CONCLUding REMARKS}

In Sloan and Lyness [6], we introduced a classification of lattice rules based on the set of invariants $n_{1}, n_{2}, \ldots, n_{s}$. In this paper, for projection regular rules only, we have extended the argument to obtain a unique categorization, based on a standard form of the associated $Z$-matrix.

It seems likely that the class of projection regular rules is wide enough to contain many interesting rules. However, not all rules are projection regular, even after an interchange of coordinate axes. For example, the 42-point 3dimensional rank-1 rule

$$
Q f=\frac{1}{42} \sum_{j=1}^{42} \bar{f}\left(j \frac{(2,3,16)}{42}\right)
$$


has no one-dimensional projections of order 42 , and so cannot be projection regular, even after interchange.

It is interesting to note that the search for good lattice rules of rank 1 by Maisonneuve [2] was in fact restricted to projection regular rules, through the first component of $\mathbf{z}=\mathbf{z}_{1}$ being forced to have the value 1. Her list (Maisonneuve [2]) includes optimal rank-1 rules, i.e., the most economic rule (or rules) having a particular Zaremba $\rho$ index (Zaremba [7]). A brief check by the present authors showed that in the range $10<N<150$ with $s=3$ she found all of the projection regular rules of this form. If all rank-1 rules are allowed, then the rule (6.1) above should replace the one with $N=44$ on her list, since both have $\rho=6$ and (6.1) is more economic. The rest of Maisonneuve's results in this range would be unchanged by the wider search.

\section{APPENDIX}

Lemma A1. Let $l, m, n$ be integers, with $n>0$. There exists an integer $k$ such that

$$
(l+k m, m)=(l, m, n) .
$$

Proof. Since the result is trivial if $l$ or $m$ is zero, we may assume $l, m$ nonzero. It is sufficient to prove the result for the case $(l, m, n)=1$, since if $(l, m, n)=a$ we may apply that result to $l^{\prime}=l / a, m^{\prime}=m / a, n^{\prime}=n / a$.

Given $(l, m, n)=1$, we are required to choose $k$ so that $(l+k m, n)=1$. Let $p_{i}$ be the $i$ th prime, and write $l, m, n$ as prime power decompositions,

$$
l= \pm \prod p_{i}^{\lambda_{i}}, \quad m= \pm \prod p_{i}^{\mu_{i}}, \quad n=\prod p_{i}^{\nu_{i}} .
$$

Since $(l, m, n)=1$, it follows that for each prime $p_{i}$ at least one of $\lambda_{i}, \mu_{i}$, $\nu_{i}$ is zero. Defining

$$
\alpha_{i}= \begin{cases}1 & \text { if } \nu_{i} \neq 0, \lambda_{i}=\mu_{i}=0 \\ 0 & \text { otherwise }\end{cases}
$$

we may choose

$$
k=\prod p_{i}^{\alpha_{i}} .
$$

Then it is straightforward to verify that any prime $p_{i}$ which divides $n$ does not divide $l+\mathrm{km}$, since it divides one term but not the other.

\section{ACKNOWLEDGMENT}

We are grateful to the Australian Research Council and the U. S. Department of Energy for generous financial support. 


\section{BIBLIOGRAPHY}

1. J. N. Lyness and I. H. Sloan, Some properties of rank-2 lattice rules, Math. Comp. 53 (1989), 627-637.

2. D. Maisonneuve, Recherche et utilisation des 'bons treillis'. Programmation et résultats numériques, in Applications of Number Theory to Numerical Analysis (S. K. Zaremba, ed.), Academic Press, London, 1972, pp. 121-201.

3. H. Niederreiter, Quasi-Monte Carlo methods and pseudo-random numbers, Bull. Amer. Math. Soc. 84 (1978), 957-1041.

4. I. H. Sloan, Lattice methods for multiple integration, J. Comput. Appl. Math. 12, 13 (1985), 131-143.

5. I. H. Sloan and P. J. Kachoyan, Lattice methods for multiple integration: theory, error analysis and examples, SIAM J. Numer. Anal. 24 (1987), 116-128.

6. I. H. Sloan and J. N. Lyness, The representation of lattice quadrature rules as multiple sums, Math. Comp. 52 (1989), 81-94.

7. S. K. Zaremba, La méthode des 'bons treillis' pour le calcul des intégrales multiples, in Applications of Number Theory to Numerical Analysis (S. K. Zaremba, ed.), Academic Press, London, 1972, pp. 39-119.

School of Mathematics, University of New South Wales, Sydney, New South Wales 2033, AUSTRALIA. E-mail: munnari!hydra.maths.unsw.oz!sloan@seismo.css.gov

Mathematics \& Computer Science Division, Argonne National laboratory, Argonne, ILLINOIs 60439-4801. E-mail: lyness@mcs.anl.gov 